\title{
H.E.S.S. discovery of very-high-energy gamma-ray emission of PKS 1440-389
}

\section{H. Prokoph ${ }^{* a}$, Y. Becherini ${ }^{a}$, M. Böttcher ${ }^{b}$, C. Boisson ${ }^{c}$, J.-P. Lenain ${ }^{d}$, and I. Sushch ${ }^{b}$ for the H.E.S.S. Collaboration}

${ }^{a}$ Department of Physics and Electrical Engineering, Linnaeus University, 35195 Växjö, Sweden

${ }^{b}$ Centre for Space Research, North-West University, Potchefstroom 2520, South Africa

${ }^{c}$ LUTH, Observatoire de Paris, CNRS, Université Paris Diderot, 5 Place Jules Janssen, 92190

Meudon, France

${ }^{d}$ LPNHE, Université Pierre et Marie Curie Paris 6, Université Denis Diderot Paris 7,

CNRS/IN2P3, 4 Place Jussieu, F-75252, Paris Cedex 5, France

E-mail: heike.prokophelnu.se

\begin{abstract}
Blazars are the most abundant class of known extragalactic very-high-energy (VHE, E > $100 \mathrm{GeV}$ ) gamma-ray sources. However, one of the biggest difficulties in investigating their VHE emission resides in their limited number, since less than 60 of them are known by now.

In this contribution we report on H.E.S.S. observations of the BL Lac object PKS 1440-389. This source has been selected as target for H.E.S.S. based on its high-energy gamma-ray properties measured by Fermi-LAT. The extrapolation of this bright, hard-spectrum gamma-ray blazar into the VHE regime made a detection on a relatively short time scale very likely, despite its uncertain redshift. H.E.S.S. observations were carried out with the 4-telescope array from February to May 2012 and resulted in a clear detection of the source. Contemporaneous multi-wavelength data are used to construct the spectral energy distribution of PKS 1440-389 which can be described by a simple one-zone synchrotron-self Compton model.
\end{abstract}

The 34th International Cosmic Ray Conference,

30 July- 6 August, 2015

The Hague, The Netherlands

\footnotetext{
* Speaker.
} 


\section{Introduction}

Active galactic nuclei (AGN), in particular blazars, are the most abundant extragalactic objects detected at very high energies (VHE; $E>100 \mathrm{GeV}$ ), constituting a third of the known VHE gamma-ray sources. Due to their fast flux decrease with increasing energy, observations at VHE are mostly performed with ground-based imaging atmospheric Cherenkov telescopes (IACTs). The small field of view of current IACTs makes new source discoveries from a large-scale sky survey difficult and therefore the search for VHE-emitting blazars has historically involved targeted observations of source candidates selected based on their radio and X-ray spectra [1]. With the launch of Fermi in June 2008, this has partly shifted towards a selection based on extrapolations of highenergy (HE; $100 \mathrm{MeV}<E<100 \mathrm{GeV}$ ) gamma-ray spectra into the VHE regime [2]. This lead to several new blazar discoveries and motivated the choice for targeted observations of PKS 1440-389 with H.E.S.S. and, eventually, its VHE discovery in April 2012 [3].

PKS 1440-389 (R.A. $=14^{h} 43^{m} 57^{s}$, decl. $=-39^{\circ} 08^{\prime} 39^{\prime \prime}$, J2000 [4]) is a bright blazar in the Fermi-LAT energy regime and has a hard, well-constrained HE spectrum (spectral index: $\Gamma_{2 \mathrm{FGL}}=$ $1.77 \pm 0.06$ [5]). Its distance was estimated as $z=0.065$ (6dF Galaxy Survey [6]), but this redshift value is not included anymore in the final version of the $6 \mathrm{dF}$ catalog due to poor spectral quality [7]. Despite many measurements in different wavelength ranges, the redshift of PKS 1440-389 remains unknown with the currently best constraint to be $0.14<z<2.2$ [8].

\section{H.E.S.S. data analysis and results}

H.E.S.S. is an array of five imaging atmospheric Cherenkov telescopes located in the Khomas Highland in Namibia which is sensitive to gamma-ray energies from a few tens of $\mathrm{GeV}$ to about $100 \mathrm{TeV}$ [9]. Observations presented here were taken before the installation of the fifth telescope for which the array characteristics are given in [10].

Observations of PKS 1440-389 were taken between 29 Feb and 27 May 2012 at a mean zenith angle of 17 degrees. All data were taken in wobble mode, for which the source is observed with an offset of $0.5^{\circ}$ with respect to the center of the instrument's field of view to allow for simultaneous background measurements [11]. After quality selection and dead time correction, the data sum up to a total live time of about 12 hours. This data set was analyzed with pre-defined cuts, optimized for a steep-spectrum source as decribed in $[12]^{1}$. The signal was extracted using a reflected region background model with an $\mathrm{ON}$ region of $0.11^{\circ}$ radius centered on the position of PKS 1440-389. In this ON region, 183 excess events have been detected, corresponding to a significance of $9.1 \sigma$ using Eq. 17 in [14]. The excess is found to be consistent with a point-like source and the corresponding sky map is shown in Figure 1. A fit to the uncorrelated excess map yields a position for the excess consistent with the radio position of PKS 1440-389 [4].

The differential energy spectrum of the gamma-ray emission was derived using a forwardfolding maximum likelihood fit [15]. The photon spectrum, shown in Figure 2, is well-described by a power-law function ${ }^{2}$ with index $\Gamma=3.61 \pm 0.34$ and a flux normalization of $N_{0}=(7.98 \pm$ $1.22) \times 10^{-12} \mathrm{~cm}^{-2} \mathrm{~s}^{-1} \mathrm{TeV}^{-1}$ at the decorrelation energy of $E_{0}=418 \mathrm{GeV}$ (statistical errors only).

\footnotetext{
${ }^{1}$ Results were cross-checked with an independent analysis package [13], yielding compatible results.

${ }^{2} d N / d E=N_{0} \cdot\left(E / E_{0}\right)^{-\Gamma}$
} 

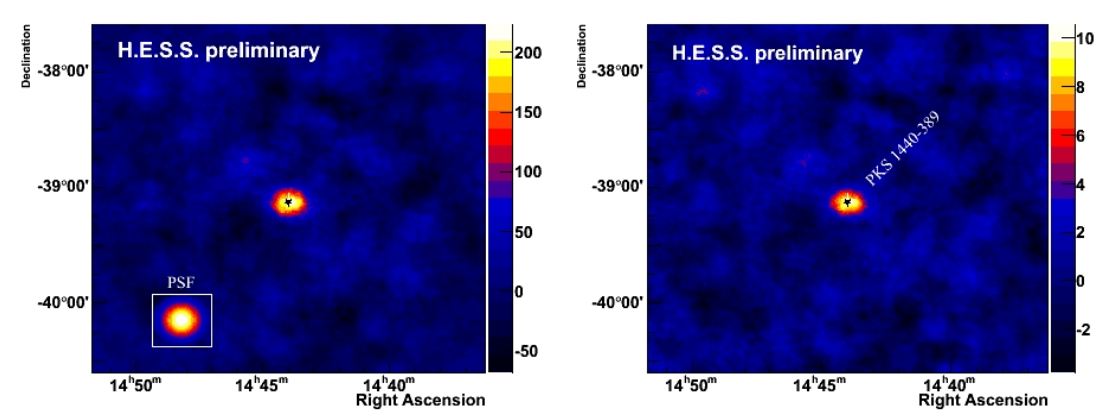

Figure 1: H.E.S.S. sky maps around the position of PKS 1440-389 (marked with a star). (Left) The excess map is smoothed with a Gaussian with a width corresponding to the $68 \%$ confinement radius of the point spread function (PSF) for this analysis (shown inline). (Right) Corresponding H.E.S.S. significance map.

The integral flux above $220 \mathrm{GeV}$ is $(6.81 \pm 1.04) \times 10^{-12} \mathrm{~cm}^{-2} \mathrm{~s}^{-1}$, corresponding to about $3 \%$ of the Crab Nebula flux [10] above the same energy threshold. A daily binned light curve was derived for $E>220 \mathrm{GeV}$ and is shown in Figure 3. A constant fit to these flux points showed no significant deviation from a steady flux $\left(\chi^{2} / n d f=19.41 / 14\right)$.

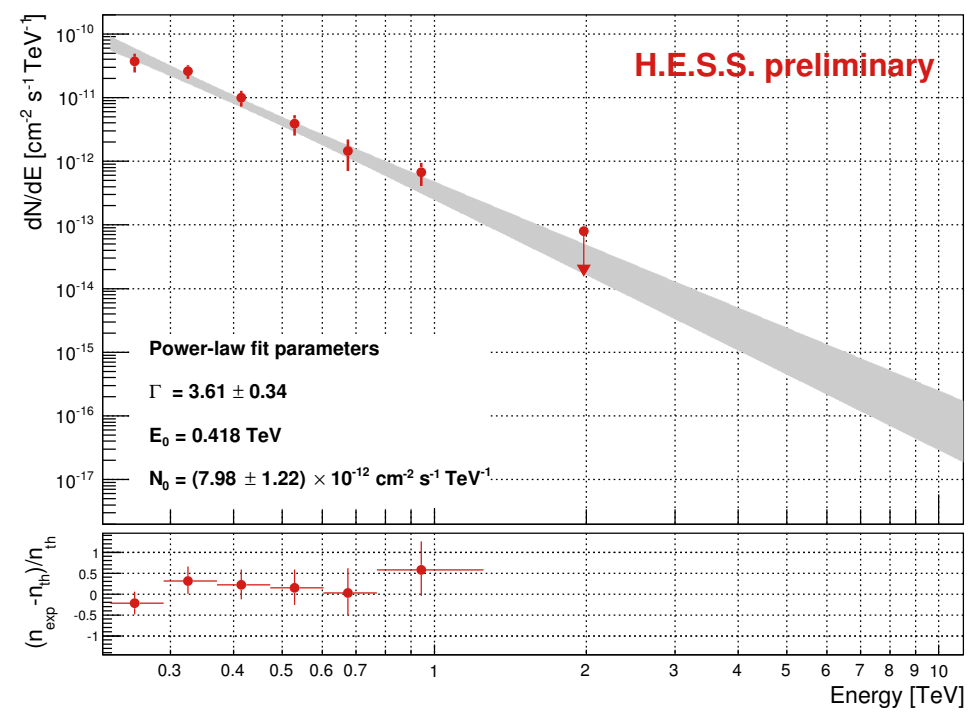

Figure 2: Differential energy spectrum of the VHE gamma-ray emission of PKS 1440-389. Overlaid spectral points were rebinned, requiring a minimum point significance of two sigma per bin.

\section{Multiwavelength data analysis and results}

\subsection{Fermi/LAT}

The Large Area Telescope (LAT) on board the Fermi satellite is a pair-conversion gammaray detector, sensitive in the energy range from $20 \mathrm{MeV}$ to $>300 \mathrm{GeV}$ [16]. Data from Aug 2008 to Feb 2015 were analyzed using the LAT ScienceTools (version v9r33p0) together with the P7REP_SOURCE_V15 instrument response functions. An unbinned likelihood analysis was 
applied to photon events with energies from $300 \mathrm{MeV}$ to $300 \mathrm{GeV}$ which were selected in a circular region of $10^{\circ}$ radius centered on the position of PKS 1440-389, resulting in an excess of about $49 \sigma$ significance. Two spectral models were applied: a power law and a log-parabola. The results are summarized in Table 1. Using a likelihood ratio test, the log-parabola model was preferred with respect to the power-law model at the $4.1 \sigma$ level. A three-monthly binned light curve for the full time range was computed, resulting in a probability of about $3.4 \%$ for a constant fit $\left(\chi^{2} / n d f=\right.$ $39.33 / 25)$. The calculation of the excess variance [17] showed only a small hint for variability $\left(F_{v a r}=0.13 \pm 0.06\right)$ in the HE regime. However, no evidence for variability on a monthly time scale has been seen within the data set contemporaneous with the H.E.S.S. observations, shown in Figure 3.

Table 1: Results of the spectral fit to the Fermi-LAT data.

\begin{tabular}{ccccc}
\hline \hline Model & $\alpha$ & $\beta$ & TS & $\begin{array}{c}\text { Flux }(0.3-300 \mathrm{GeV}) \\
{\left[10^{-9} \mathrm{ph} \mathrm{cm}^{-2} \mathrm{~s}^{-1}\right]}\end{array}$ \\
\hline Log-parabolic (LP) & $1.32 \pm 0.04$ & $0.083 \pm 0.008$ & 2403.9 & $8.42 \pm 0.42$ \\
Power law (PL) & $1.79 \pm 0.03$ & - & 2411.8 & $9.95 \pm 0.53$ \\
\hline
\end{tabular}

\subsection{Swift/XRT and UVOT}

The X-ray telescope (XRT) on board the Swift satellite is designed to measure X-rays in the $0.2-10 \mathrm{keV}$ energy range [18]. Target of opportunity observations were obtained on 2012 April 29 (MJD 56046), following the VHE discovery of PKS 1440-389. The analysis of this $8 \mathrm{ks}$ exposure was performed using standard tools (HEASoft 6.16, Xspec 12.8.2). Data were grouped, requiring a minimum of 20 counts per bin, and then fitted with a power-law model. Photo-electric absorption was fit using the Wisconsin cross-section [19] with a fixed value for the Galactic column density of $N_{\text {tot }}=1.08 \times 10^{21} \mathrm{~cm}^{-2}$ [20], resulting in a photon index of $\Gamma=2.64 \pm 0.05$ and $N_{0}=(3.80 \pm$ $0.12) \times 10^{-3} \mathrm{~cm}^{-2} \mathrm{~s}^{-1} \mathrm{keV}^{-1}$ at $1 \mathrm{keV}$. It should be noted, that when $N_{\text {tot }}$ was left free during the fit, a slightly better fit could be obtained with a column density compatible with the total value.

Simultaneously with the XRT observations, the Swift Ultra Violet and Optical Telescope (UVOT [21]) carried out observations in all six filters. Sky-corrected images were taken from the Swift archive, and analysis was performed using the uvotmaghist, uvotimsum and uvot source tasks included in the FTOOLS software package. Source counts were extracted using a 5 " radius for V, B and U filters and a 12" for the UVW1, UVM2 and UVW2 filters. The background was extracted from source-free regions in the surroundings. Filter UVW1 and UVM2 observations having four individual exposures, these were stacked prior to aperture photometry as the uvotmaghist task showed no variability between the individual exposures. Count rates are then converted to fluxes using the standard photometric zero points [22]. The reported fluxes are de-reddened for Galactic absorption following the description in [23], with $E(B-V)=0.103$ mag.

\section{3 АTOM}

The Automatic Telescope for Optical Monitoring (ATOM) is a fully automatic $75 \mathrm{~cm}$ optical telescope operated by LSW Heidelberg on the H.E.S.S. site [24]. Operating since 2005, ATOM 


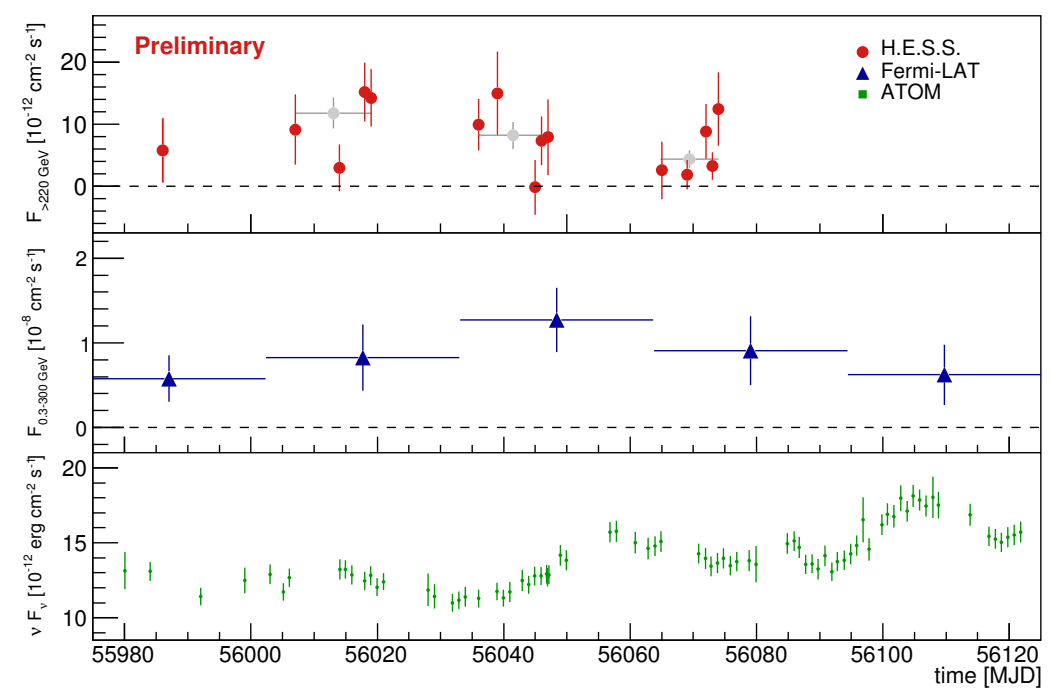

Figure 3: Light curve of PKS 1440-389 in different wavelength regimes. (top) Nightly (red) and monthly (gray) binned fluxes above $220 \mathrm{GeV}$ measured by H.E.S.S. (middle) Monthly binned fluxes measured by Fermi-LAT. (bottom) Nightly optical fluxes measured by ATOM in the R band.

provides optical monitoring of gamma-ray sources and has observed PKS 1440-389 with high cadence in 2012 in the R band. Following the VHE discovery, additional B, V and J band observations were obtained. The ATOM data were analysed using the Automatic Data Reduction and Analysis Software (ADRAS) which automates standard data reduction and performs differential photometry using five known nearby sources as calibrators. A preliminary light curve is presented in Figure 3, showing clear variability in the optical regime contemporaneous with the H.E.S.S. observations.

\section{Spectral energy distribution and modeling}

A spectral energy distribution (SED) of PKS 1440-389 was derived using the above described multi-wavelength data sets. As no significant variability was detected in the gamma-ray regimes, the spectra resulting from the analyses of the full data sets were used. Additionally, archival data from the 2MASS [25] and WISE catalogs [26] were used to complete the low-energy part of the SED.

The overall, non-simultaneous SED of PKS 1440-389 is shown in Figure 4. It exhibits the usual two bumps seen in blazars, one at low frequencies, from radio to $\mathrm{X}$-rays, and the other at higher frequencies. This SED was modeled with a one-zone leptonic model [27], taking the extragalactic absorption into account [28]. Within the model the low-energy emission is interpreted as synchrotron emission from relativistic electrons in a spherical emission region, moving relativistically along the jet which is closely aligned with the line-of-sight. The high-energy gamma-ray emission is produced via Compton up-scattering off the same electron population which produced the synchrotron peak (synchrotron-self Compton; SSC). The non-thermal electron distribution in the emission region is determined self-consistently as an equilibrium between injection of a powerlaw distribution, radiative cooling, and particle escape. Due to the uncertainty in the distance to 
PKS 1440-389, the SED modeling was done for two redshifts ${ }^{3}: z=0.065$ and $z=0.14$. The results are shown in Figure 4. It can be seen that the overall SED is moderately well described by the model for the used parameter values which are given in Table 2. The parameter values themselves are well within the range of those used for previously detected VHE blazars [29] with a relatively low magnetic field strength and a large emission region; resulting in a particle dominated jet $\left(L_{B} / L_{e}<0.01\right.$, $L_{B}$ and $L_{e}$ being the magnetic and kinetic jet power, respectively). Models with additional external radiation fields yield, in general, jet energetics closer to equipartition $\left(L_{B} / L_{e} \sim 1\right)$, but due to the lack of additional observational constrains, applying such a more complex model to the SED of PKS 1440-389 would result in strongly underconstrained parameters and seems therefore not justified.

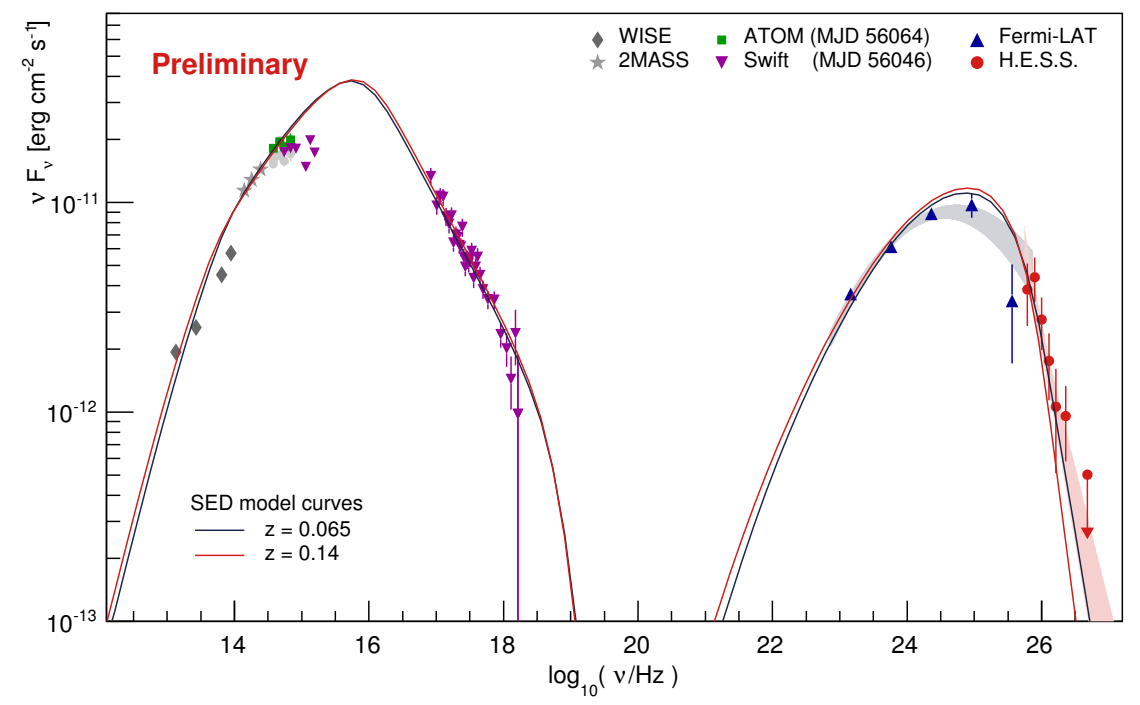

Figure 4: Spectral energy distribution of PKS 1440-389 together with the one-zone SSC model for two possible redshifts (see text for details).

\begin{tabular}{|c|c|c|c|c|c|c|c|c|c|c|}
\hline$z$ & $\begin{array}{c}L_{e} \\
{\left[10^{44} \mathrm{erg} / \mathrm{s}\right]}\end{array}$ & $\begin{array}{c}\gamma_{1} \\
{\left[10^{3}\right]}\end{array}$ & $\begin{array}{c}\gamma_{2} \\
{\left[10^{5}\right]}\end{array}$ & $q$ & $\begin{array}{c}B \\
{[\mathrm{G}]}\end{array}$ & $\begin{array}{c}R \\
{\left[10^{16} \mathrm{~cm}\right]}\end{array}$ & $\Gamma$ & $\eta_{\mathrm{esc}}$ & $\varepsilon_{B e}$ & $\begin{array}{l}\delta t_{\mathrm{var}} \\
{[\mathrm{hr}]}\end{array}$ \\
\hline 0.065 & 0.426 & 72 & 20 & 3.2 & 0.02 & 12 & 10 & 50 & 0.00507 & 86 \\
\hline 0.14 & 1.038 & 78 & 20 & 3.2 & 0.02 & 27 & 10 & 25 & 0.01054 & 207 \\
\hline
\end{tabular}

Table 2: SSC model parameters for PKS 1440-389. The columns are the following: $z$ is the assumed redshift; $L_{\mathrm{e}}$ is the (kinetic) jet luminosity; $\gamma_{1}$ is the low energy cutoff energy of the electron distribution; $\gamma_{2}$ is the high energy cutoff; $q$ is the spectral index of the electron injection spectrum; $B$ is the magnetic field strength; $R$ is the emission region radius; $\Gamma$ is the bulk Lorentz factor; and $\eta_{\text {esc }}$ is the escape time parameter with $t_{\mathrm{esc}}=\eta_{\mathrm{esc}} \cdot R / c$. Additionally, two output parameters are given: $\varepsilon_{B e}$ is the resulting relative partition parameter $\varepsilon_{B e}=L_{\mathrm{B}} / L_{\mathrm{e}}$; and $\delta t_{\mathrm{var}}$ is the resulting minimum variability time scale.

\footnotetext{
${ }^{3}$ Redshifts are converted to luminosity distances using a $\Lambda$ CDM cosmology with $H_{0}=70 \mathrm{~km} \mathrm{~s}^{-1} \mathrm{Mpc}^{-1}, \Omega_{\mathrm{m}}=0.3$ and $\Omega_{\Lambda}=0.7$.
} 


\section{Summary and Conclusions}

In this paper, we reported on the H.E.S.S. discovery of the BL Lac object PKS 1440-389 in 2012. The source was selected as VHE blazar candidate based on its hard high-energy spectrum measured by Fermi-LAT, but is also listed in [30] based on its infrared and X-ray properties. The average flux in the VHE regime was about 3\% of the Crab Nebula flux and showed no significant gamma-ray variability. Multi-wavelength data, contemporaneous with the H.E.S.S. observations, were used to derive an SED which is well reproduced with a one-zone leptonic model with parameters typical for other VHE blazars.

Acknowledgments: The support of the Namibian authorities and of the University of Namibia in facilitating the construction and operation of H.E.S.S. is gratefully acknowledged, as is the support by the German Ministry for Education and Research (BMBF), the Max Planck Society, the German Research Foundation (DFG), the French Ministry for Research, the CNRS-IN2P3 and the Astroparticle Interdisciplinary Programme of the CNRS, the U.K. Science and Technology Facilities Council (STFC), the IPNP of the Charles University, the Czech Science Foundation, the Polish Ministry of Science and Higher Education, the South African Department of Science and Technology and National Research Foundation, and by the University of Namibia. We appreciate the excellent work of the technical support staff in Berlin, Durham, Hamburg, Heidelberg, Palaiseau, Paris, Saclay, and in Namibia in the construction and operation of the equipment.

\section{References}

[1] Costamante, L., \& Ghisellini, G., TeV candidate BL Lac objects, A\&A 384, 56 (2002) [arXiv:astro-ph/0112201]

[2] Tavecchio, F., Ghisellini, G., Ghirlanda, G., et al., TeV BL Lac objects at the dawn of the Fermi era, MNRAS 401, 3 (2010) [arXiv: 0909.0651 ]

[3] Hofmann, W. et al., Very high energy gamma-ray emission detected from PKS 1440-389 with H.E.S.S., The Astronomer's Telegram 4072, 1 (2012)

[4] Jackson, C. A., Wall, J. V., Shaver, P. A., et al., The Parkes quarter-Jansky flat-spectrum sample. I. Sample selection and source identifications, A\&A 386, 97 (2002)

[5] Nolan, P. L., Abdo, A. A., Ackermann, M., et al., Fermi Large Area Telescope Second Source Catalog, The Astrophysical Journal Supplement 199, 31 (2011) [arXiv:1108.1435]

[6] Jones, D. H., Saunders, W., Colless, M., et al., The 6dF Galaxy Survey: samples, observational techniques and the first data release, MNRAS 355, 3 (2004) [arXiv: astro-ph/ 0403501 ]

[7] Jones, D. H., Read, M. A., Saunders, W., et al., The 6dF Galaxy Survey: final redshift release (DR3) and southern large-scale structures, MNRAS 399, 2 (2009) [arXiv: 0903.5451 ]

[8] Shaw, M. S., Romani, R. W., Cotter, G., et al., Spectroscopy of the Largest Ever Gamma-Ray-Selected BL Lac Sample, ApJ 764, 135 (2013) [arXiv: 1301 . 0323]

[9] De Naurois, M. et al., H.E.S.S. II Highlights - Gamma-ray astronomy from $20 \mathrm{GeV}$ to $100 \mathrm{TeV}$, in proceeding of ICRC 2015

[10] Aharonian, F., Akhperjanian, A. G., Bazer-Bachi, A. R., et al., Observations of the Crab nebula with H.E.S.S., A\&A 457, 899 (2006) [arXiv: astro-ph/ 0607333 ] 
[11] Fomin, V. P., Fennell, S., Lamb, R.C., et al., New methods of atmospheric Cherenkov imaging for gamma-ray astronomy. II. The differential position method, Astroparticle Physics 2, 137 (1994)

[12] Becherini, Y., Djannati-Atai, A., Marandon, V., et al., A new analysis strategy for detection of faint gamma-ray sources with Imaging Atmospheric Cherenkov Telescopes, Astroparticle Physics 34, 858 (2011) [arXiv:1104 .535]

[13] De Naurois, M. \& Rolland, L., A high performance likelihood reconstruction of gamma-rays for imaging atmospheric Cherenkov telescopes, Astroparticle Physics 32, 231 (2009) [arXiv:0907.2610]

[14] Li, T.-P. \& Ma, Y.-Q., Analysis methods for results in gamma-ray astronomy, ApJ 272, 317 (1983)

[15] Piron, F., Djannati-Atai, A., Punch, M., et al., Temporal and spectral gamma-ray properties of Mkn 421 above $250 \mathrm{GeV}$ from CAT observations between 1996 and 2000, A\&A 374, 895 (2001) [arXiv:astro-ph/0106196]

[16] Atwood, W.B, Abdo, A.A., Ackermann, M., et al., The Large Area Telescope on the Fermi Gamma-Ray Space Telescope Mission, ApJ 697, 1071 (2009) [arXiv: 0902 . 1089]

[17] Vaughan, S., Edelson, R., Warwick, R. S., et al., On characterizing the variability properties of X-ray light curves from active galaxies, MNRAS 345, 1271 (2003) [arXiv: astro-ph/0307420]

[18] Burrows, D.N, Hill, J.E., Nousek, J.A., et al., The Swift X-Ray Telescope, Space Science Reviews 120, 165 (2005) [arXiv:astro-ph/0508071]

[19] Morrison, R. \& McCammon, D., Interstellar photoelectric absorption cross sections, 0.03-10 keV, ApJ 270, 119 (1983)

[20] Willingale, R., Starling, R. L. C., Beardmore, A. P., et al., Calibration of X-ray absorption in our Galaxy, MNRAS 431, 394 (2013) [arXiv: 1303 . 0843]

[21] Roming, P.W.A., Kennedy, T.E., Mason, K.O., et al., The Swift Ultra-Violet/Optical Telescope, Space Science Reviews 120, 95 (2005) [arXiv: astro-ph/ 0507413 ]

[22] Poole, T. S., Breeveld, A. A., Page, M. J., et al., Photometric calibration of the Swift ultraviolet/optical telescope, MNRAS 383, 627 (2008) [arXiv: 0708 .2259]

[23] Roming, P. W. A., Koch, T. S., Oates, S. Ret al., The First Swift Ultraviolet/Optical Telescope GRB Afterglow Catalog, ApJ 690, 163 (2009) [arXiv: 0809 . 4193]

[24] Hauser, M., Möllenhoff, C., Pühlhofer, G., et al., ATOM - an Automatic Telescope for Optical Monitoring, Astronomische Nachrichten 325, 659 (2004)

[25] Mao, L. S., 2MASS observation of BL Lac objects II, New Astronomy 16, 503 (2011)

[26] Wright, E. L., Eisenhardt, P. R. M., Mainzer, A. K., et al., The Wide-field Infrared Survey Explorer (WISE): Mission Description and Initial On-orbit Performance, AJ 140, 1868 (2010) [arXiv: 1008 .0031]

[27] Böttcher, M., Reimer, A., Sweeney, K., \& Prakash, A., Leptonic and Hadronic Modeling of Fermi-detected Blazars, ApJ 768, 54 (2013) [arXiv: 1304.0605 ]

[28] Finke, J.D., Razzaque, S., and Dermer C.D., Modeling the Extragalactic Background Light from Stars and Dust, ApJ 712, 238 (2010) [arXiv:0905.1115]

[29] Aliu, E., Archambault, S., Arlen, T., et al., Long term observations of B2 1215+30 with VERITAS, ApJ 779, 92 (2013) [arXiv:1310.6498]

[30] Massaro, F., Paggi, A., Errando, M., et al., BL Lac Candidates for TeV Observations, ApJS 207, 16 (2013) [arXiv: 1307.8113$]$ 